\title{
Genomics contest underscores challenges of personalized medicine
}

On a clear, cold afternoon in December 2010, a crowd of industry and academic leaders gathered in the Countway Library of Medicine at the Harvard Medical School in Boston to discuss one of the most pressing concerns in medicine: how to tame the expected deluge of 'whole genome' sequence data into clinically useful information. For personalized medicine to become commonplace, the field needed a series of tools and standards for genomic analysis and interpretation, everyone agreed. But these didn't exist yet. "Researchers were going about it one way, and clinicians were going about it another," says Alan Beggs, director of the Manton Center for Orphan Disease Research at the Children's Hospital Boston who helped organize the event.

The result of the meeting was the creation of the CLARITY Challenge, a competition launched this past January by Beggs and his Children's Hospital colleagues. Short for 'Children's Leadership Award for the Reliable Interpretation and appropriate Transmission of Your genomic information', the $\$ 25,000$ contest aims to encourage academic and commercial groups to develop the best methods-including databases, software and clinical reports-to discover the unknown genetic basis of inherited pediatric disorders and then communicate that information in a way that can be understood by general practitioners and affected families. The winning prize will go to the team that can successfully analyze the genome sequences of three children with mysterious hereditary illnesses and pro-

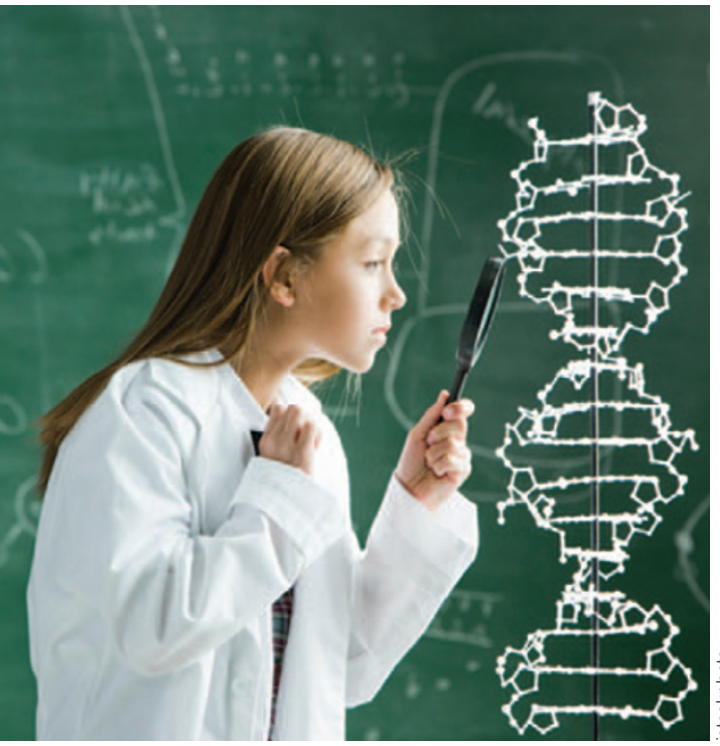

No kidding: Genomes ares tough to interpret. duce the most clinically useful reports. The contest will kick off next month with up to 20 teams, and the winner will be announced in October.

Beggs acknowledges that the prize money isn't enough to fund the actual research, but he hopes that it adds an incentive to the challenge. "It's more the prestige and hopefully the publicity" of winning that will attract researchers to apply, he says.

Investigators in the field welcome the initiative. "Digesting all of the findings in a genome and presenting them back to the clinician and patient in an orderly and understandable fashion-that, in my mind, is the holy grail," says Howard Levy, a clinical geneticist at the Johns Hopkins University School of Medicine in Baltimore. "I love the idea that rather than just pounding the drum and saying, 'Someone needs to do this,' they're stepping forward and incentivizing it."

For contestants, the sequencing of a genome is the easy part. The rest of the process-sifting through 3 billion base pairs for 'actionable' genetic variants and then making that information digestible for doctorsis the challenge. "The biggest bottleneck is that we're spending so much money discovering [genetic variants] and not enough money actually testing their clinical utility," says Jonathan Berg, a medical geneticist at the University of North Carolina School of Medicine in Chapel Hill.

Once clinically relevant genes are identified, researchers and physicians then need fast and easy ways to share and store the massive amounts of genetic information, says Daniel Masys, who studies biomedical and health informatics at the University of Washington in Seattle. To that end, Masys advocates tossing out the bulk of people's DNA data and recording only the differences between individual genomes and a national reference sequence. In a study published online in December, Masys and his colleagues found that implementing such a strategy would mean that researchers could store just $1 \%$ of all the data without sacrificing any useful information (J. Biomed. Inform. doi:10.1016/j.jbi.2011.12.005, 2011). "If the genomic data [for each person] is $96-99 \%$ the same, then why store it all?" he asks.

Finally, scientists have to find ways of communicating the relevant findings to physicians and their patients. "We don't want to end up sending an Excel spreadsheet to a clinician," says Shashikant Kulkarni, medical director of genomics and pathology services at Washington University School of Medicine in St. Louis.

\section{Bin there, done that}

Even before the competition launched, scientists had begun offering solutions to this problem. Last year, for instance, Berg and his colleagues proposed a 'binning' technique for classifying genetic variants according to their clinical usefulness. Under Berg's scheme, researchers would place variants into one of three bins: those that are immediately actionable, those associated with a condition but for which there are no current treatments and those with unknown clinical significance (Genet. Med. 13, 499-504, 2011). Doctors would probably then be obligated to share the information in the first category, but patients could decide whether they wanted to know about other kinds of variants. The bins would be regularly updated as new scientific data are generated.

"This is a practical attempt to carve up the genome in a way that makes it manageable and tractable," Berg says. His team has recently begun conducting a four-year study to see how the binning model works in actual clinical practice.

Even once all the tools are in place, primary-care doctors will still need some sort of genetics reference guide to understand genomic results. A 2008 study by Levy and his colleagues tested nine online resources for nongeneticists using basic questions about five common genetic conditions, and found that the websites had complete descriptions about the conditions only a third of the time and contained no information at all another third of the time (Genet. Med. 10, 659-667, 2008). In response, Levy's group, together with the US National Coalition for Health Professional Education in Genetics, recently launched a website, GeneFacts.org, to provide quick but accurate descriptions of genetic conditions for point-of-care, decision support.

No matter what approach is taken, contestants in the CLARITY Challenge have their work cut out for them. And although it will certainly take more than one contest to bring genomics to the clinic, experts agree that it's only a matter of time. "There's no question that we're going to get there," says Jeffrey Saffitz, chair of the pathology department at the Beth Israel Deaconess Medical Center in Boston. "But between now and then, it's probably going to be kind of messy."

Megan Scudellari 